\title{
Relación del nivel de hidratación y la pérdida de masa grasa en personas que realizan actividad física en gimnasios de la Urbanización Alameda de Ñaña, Lurigancho Chosica, Lima 2013
}

\author{
Relationship of hydration and fat mass loss in people who exercise in gyms Alameda Urbanization, \\ Chosica, Lurigancho, Lima, 2013
}

Elisban Vicente Centón Quispeㄹ, Elí Pedro Pérez Fernández², Mery Rodríguez Vásquezº

\begin{abstract}
RESUMEN
Objetivo: Determinar la relación entre el nivel de hidratación y la pérdida de grasa en personas que realizan actividad física en gimnasios de la urbanización La Alameda de Ñaña, Lima. Material y Métodos: Estudio descriptivo, tipo correlacional, de diseño no experimental se utilizaron equipos antropométricos como tallímetro, balanza, cinta métrica, impedanciómetro y cáliper estandarizados por el Centro Nacional de Alimentación y Nutrición (CENAN), al igual que la tabla de escala de colores Armstrong para determinar el nivel de deshidratación. La muestra fue de 80 participantes. Resultados: En los resultados referidos al nivel de hidratación ocurrió en $1.25 \%$, $85 \%$ y el $13.8 \%$ de DHT respectivamente. Además, todos los participantes redujeron su porcentaje de masa grasa. Sin embargo, se observó que en las dos primeras semanas de evaluación hubo una influencia significativa, pero en las dos restantes no hubo influencia. Conclusiones: Existe correlación significativa entre ambas variables, asimismo, existen otras variables que influyen en la pérdida de masa grasa como la alimentación, intensidad, tiempo, tipo de ejercicio, género y diferentes tipos de metabolismo.
\end{abstract}

Palabras clave: Hidratación, masa grasa, influencia, gimnasio.

\begin{abstract}
Objective: determine the relationship between the level of hydration and fat mass loss in people who exercise in gyms urbanization La Alameda, Lima. Material and Methods: Descriptive, correlational, not experimental equipment such as anthropometric measuring rod design, scale, tape measure, caliper and impedance meter, standardized by the National Center for Food and Nutrition (DINE), as table color scale used for Armstrong determining the level of dehydration. The sample consisted of 80 participants. Results: In the results related to the level of hydration occurred in $1.25 \%, 85 \%$ and $13.8 \%$ respectively of DHT. In addition, all participants reduced their percentage of body fat. However, it was observed that in the first two weeks of no significant influence evaluation and in the remaining two had no influence. Conclusions: There is significant correlation between the two variables, also there are other variables that influence the loss of fat mass and power, intensity, time, type of exercise, gender and different types of metabolism.
\end{abstract}

Keywords: hydration, fat mass , influence fitness

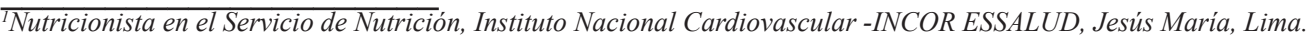
${ }^{2}$ Nutricionista, Concesionario de Alimentos MISA Corporation, Vitarte, Lima.

${ }^{3}$ Profesora Auxiliar; Coordinadora de Acreditación, E.A.P Nutrición, Facultad de Ciencias de la Salud, Universidad Peruana Unión, Lima. Correspondencia: elisban_3@hotmail.com
} 


\section{INTRODUCCIÓN}

La pérdida de líquidos tiene una influencia negativa, tanto en el rendimiento como en la integridad física del deportista, lo que pone automáticamente de relieve e importancia la sustitución de los líquidos perdidos $\mathrm{y}$, por lo tanto, de la rehidratación, problema nada sencillo, ya que se trata de escoger el momento más idóneo para cumplir las condiciones que permitan al agua y a los líquidos ingeridos ser absorbidos en el tiempo más breve posible.

En el Perú el consumo de agua es mayor para uso doméstico, de aseo, etc; sin embargo, aún no hay estudios que revelan la cantidad de agua ingerida en promedio por persona, siendo más importante aún para las personas que realizan algún tipo de actividad física (Universidad Maimonides, 2010)

Por ejemplo, en España existe El Observatorio de Hidratación y Salud, que es una institución científica y técnica que pretende mejorar los conocimientos de la hidratación y su relación con la salud, por ello promueve todo tipo de proyectos para recabar información sobre esta temática, una correcta hidratación es sinónimo de salud y como se desprende del Estudio Hábitos de Hidratación 2010, esta asociación no es percibida por la población (Tomas, 2009).

Es un hecho conocido que el consumo abundante de agua ayuda a metabolizar la grasa almacenada. Con ese fin, los riñones deben recibir una cantidad suficiente de agua. Si los riñones no están recibiendo suficiente agua, van a terminar confiando en la ayuda del hígado. Cuando el hígado está funcionando por su propia cuenta, descompone la grasa y la distribuye en forma de energía a diferentes partes del cuerpo. Cuando el hígado está sobrecargado, hace que se almacene grasa en el cuerpo en lugar de romper los enlaces. Pero al consumir agua, el hígado continuará metabolizando la grasa en lugar de almacenarlo en el cuerpo (Gil 2010; Moyano 1992).

El agua juega un papel importante en la pérdida de peso. El agua pura no contiene calorías, ni grasa, colesterol ni tampoco sodio. El agua también es un supresor del apetito, es por eso que cuanta más agua se toma, menos apetito se desarrollará. Esta es otra razón por la que el agua potable, que contiene sodio, puede potencialmente ayudar a aquellos que están tratando de perder peso (Consolazio, 1963).

Por ello, es importante realizar la evaluación del estado nutricional de las personas para contar con un diagnóstico y un seguimiento de la obesidad o desnutrición, como factor pronóstico en la evolución de múltiples procesos crónicos. Por otro lado, existe una clara relación entre algunos parámetros nutricionales y de distribución de la grasa corporal y el riesgo metabólico-cardiovascular.

Siendo el objetivo del estudio determinar la influencia del nivel de hidratación en la pérdida de masa grasa en personas que realizan actividad física en los gimnasios: Gimnasio y Salud, Aeróbicos Ruth y Club deportivo Razuri, de la Alameda, LuriganchoChosica.

\section{MATERIALES Y MÉTODOS}

El estudio se realizó en el distrito de Lurigancho, Perú, en los gimnasios de la Alameda de Ñaña: "Club Deportivo Rásuri", "Gimnasio Alameda" y Gimnasio y Aeróbicos "Ruth". Los gimnasios cuentan con aeróbicos, máquinas de cardio y de levantamiento de peso. Para realizar el estudio se obtuvo el permiso correspondiente para trabajar con todos los participantes que cumplían los criterios de inclusión del presente estudio. Siendo la muestra de 80 participantes.

\section{Técnicas e instrumentos de recolección de datos}

Para el estudio se utilizaron equipos antropométricos (tallímetro, balanza mecánica, cáliper e impedanciómetro) estandarizados por el Centro Nacional de Alimentación y Nutrición (CENAN) pertenecientes a la E.A.P. Nutrición Humana de la Universidad Peruana Unión Lima.

\section{Test de coloración de orina}

Se utilizó la prueba del test de coloración de orina, para medir la deshidratación de las personas asistentes a los gimnasios. Se tomó la primera orina del día, en un frasco de vidrio transparente para luego ser colocada en una superficie de color blanca y compararlo simultáneamente con la cartilla del test de coloración.

Además, durante el transcurso de la prueba, se utilizó la escala de colores adaptada de Armstrong para medir el nivel de coloración de orina.

Tabla de Escala de Colores Armstrong 2000:

- Técnica: Medición de la hidratación

- Material: Cartulina plastificada

- Especificaciones del Instrumento

(Tabla de escala de colores, escala de colores del 1 al 8 , lectura de diferenciación de colores rápida). 
Relación del nivel de hidratación y la pérdida de masa grasa en personas que realizan actividad física en gimnasios de la Urbanización Alameda de Ñaña, Lurigancho Chosica, Lima 2013

La muestra se sostuvo en alto frente a un fondo blanco, con buena iluminación. El color de la orina se comparará con la escala de colores de Armstrong 2000 .

Ficha de Recolección de datos

- Técnica: Ficha de recolección de datos

- Material: Hoja bond

- Especificaciones del Instrumento

Conformada por dos partes (datos generales y evaluación antropométrica)

Está dividida en cuatro períodos de evaluación y una columna de observaciones.
La ficha de recolección de datos, en la primera parte, está conformada por fecha, responsable que realiza la prueba, nombre del participante, edad, sexo, procedencia, frecuencia de entrenamiento, tiempo de entrenamiento; en la segunda parte, está conformada por peso, talla, pliegue de pecho, pliegue abdominal, pliegue de muslo, pliegue de pierna o gemelar, pliegue Submaxilar, pliegue de tríceps, pliegue de cresta Iliaca, pliegue Subescapular, IMC, \% Masa Grasa, $\%$ Masa Muscular, circunferencia cintura, coloración de orina y diagnóstico nutricional. Asimismo, la parte de la evolución antropométrica se dividió en cuatro períodos de evolución, que fue tomado durante un mes de investigación.

\section{RESULTADOS}

Tabla 1

Porcentaje del nivel de hidratación durante cuatro semanas

\begin{tabular}{ccc}
\hline Deshidratación & Número de participantes & $\%$ \\
\hline Hidratación normal & 0 & 0 \\
Deshidratación mínimo & 1 & 1.25 \\
Deshidratación significativo & 68 & 85 \\
Deshidratación severo & 11 & 13.8 \\
Total & 80 & 100 \\
\hline
\end{tabular}

En la tabla 1 se muestra que ningún participante estuvo bien hidratado. Además, durante las cuatro semanas de evaluación, se encontraron tres tipos de deshidratación, calificados como mínima, significativa y severa. El $1.25 \%$ presentó DHT mínima, el $85 \%$ DHT significativa y el $13.8 \%$ DHT severa, como se muestra en la tabla N..$^{\circ} 1$.

Tabla 2

Porcentaje de la pérdida de masa grasa en los participantes

\begin{tabular}{ccc}
\hline \% de pérdida de masa grasa & $\mathrm{N}$ & $\%$ \\
\hline-1 & 1 & 1 \\
1 & 3 & 4 \\
2 & 10 & 13 \\
3 & 15 & 19 \\
4 & 10 & 13 \\
5 & 4 & 5 \\
6 & 5 & 6 \\
7 & 9 & 11 \\
8 & 1 & 1 \\
9 & 3 & 4 \\
10 & 4 & 5 \\
11 & 4 & 5 \\
13 & 5 & 6 \\
16 & 2 & 3 \\
18 & 1 & 1 \\
20 & 1 & 1 \\
26 & 2 & 3 \\
\hline Total & 80 & 100
\end{tabular}


En la tabla 2, con respecto a la disminución de masa grasa, la mayoría de participantes que son 15 individuos se observó que un 3\% redujo masa grasa, este grupo fue evaluado sin haber recibido ninguna información sobre hidratación en caso de estar sometidos a ejercicios físicos de exigencia media, mientras que solo 2 participantes redujo el $26 \%$ de su masa grasa. Evaluados por un tiempo de cuatro semanas significativas comenzando en la primera semana con un $75 \%$ a un $78.8 \%$ en la cuarta semana.

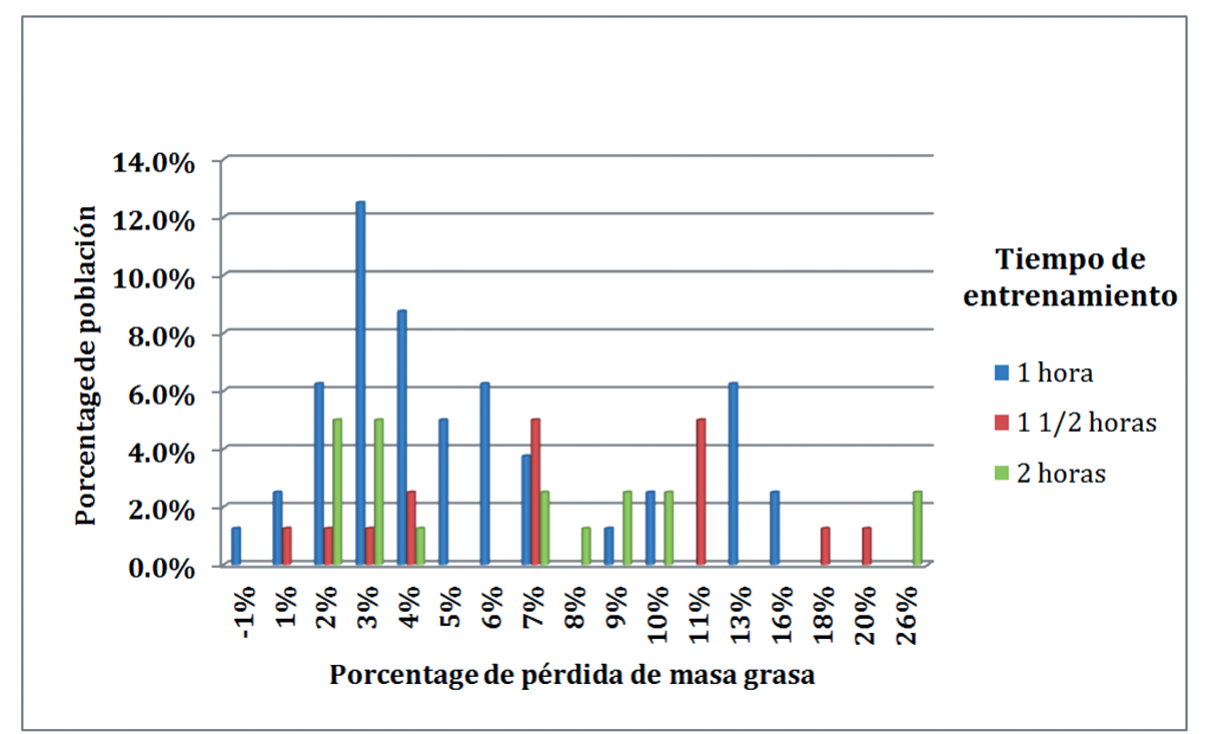

Figura 1

Tiempo de entrenamiento y porcentaje de pérdida de masa grasa

En cuanto al tiempo de entrenamiento y la pérdida de masa grasa, se muestra en la figura 1 quienes tuvieron un entrenamiento de 1 hora diaria, redujeron desde un $1 \%$ a un $16 \%$ de masa grasa, además, quienes entrenaron $1 \frac{1}{2}$ hora diaria redujeron desde un $1 \%$ a
$20 \%$ de masa grasa y aquellos que entrenaron 2 horas diarias redujeron desde un $2 \%$ a un $26 \%$ de masa grasa, y la tabla 3 nos muestra que existe una relación directa entre dichas variables, con una significancia de 0.001 .

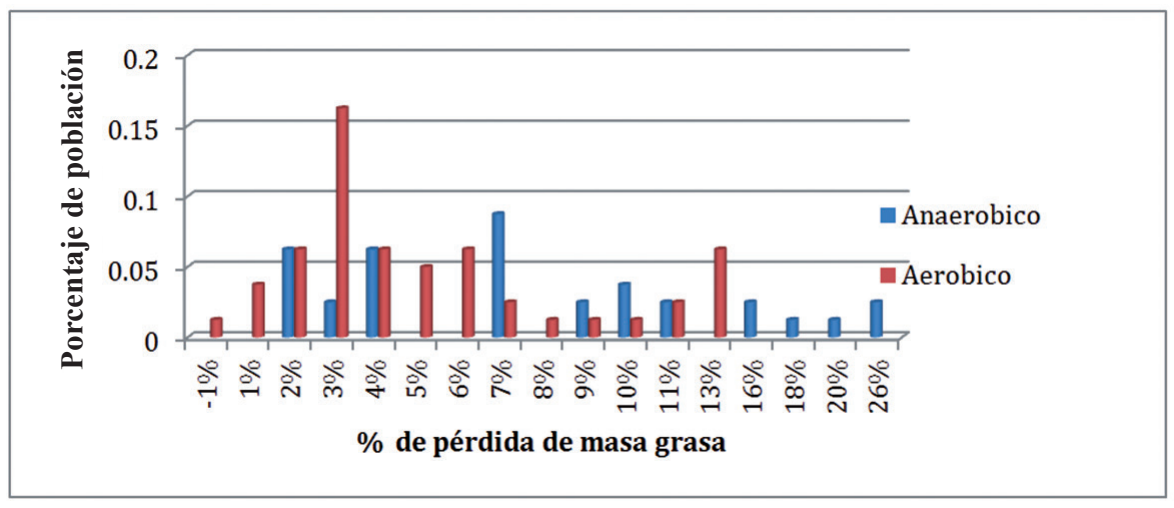

Figura 2

Tipo de entrenamiento y el porcentaje de masa grasa

Asimismo, en cuanto al tipo de entrenamiento y la pérdida de masa grasa, se muestra en el gráfico 8 , puesto que quienes realizaban actividad física anaeróbica redujeron desde un $2 \%$ a un $26 \%$ de masa grasa, mientras que aquellos quienes practicaron actividad física aeróbica redujeron entre un 1\% y $13 \%$ de masa grasa, y la tabla 4 nos muestra que existe una relación entre dichas variables, con una significancia de 0.004 . 
Relación del nivel de hidratación y la pérdida de masa grasa en personas que realizan actividad física en gimnasios de la Urbanización Alameda de Ñaña, Lurigancho Chosica, Lima 2013

Tabla 3

Relación del nivel de hidratación y el porcentaje de la pérdida de masa grasa

\begin{tabular}{cccc}
\hline & & Coloración de orina & Porcentaje de masa grasa \\
\hline \multirow{2}{*}{ Coloración de orina } & Pearson Correlación & 1 & $-0,244^{*}$ \\
& Sig. (2-tailed) & & 0,030 \\
& $\mathrm{n}$ & 80 & 80 \\
Porcentaje de masa grasa & Pearson Correlación & $-0,244^{*}$ & 1 \\
& Sig. (2-tailed) & 0,030 & \\
& $\mathrm{n}$ & 80 & 80 \\
\hline
\end{tabular}

*. Correlation is significant at the 0.05 level (2-tailed).

En la tabla 3 la relación entre el nivel de hidratación y la pérdida de masa grasa, durante las cuatro semanas de evaluación, tanto de hombres y mujeres, presentó una significancia de 0,030. La hipótesis alterna es aceptada mientras dicho valor se acerque más a cero. Esto quiere decir que se acepta la hipótesis alterna, existiendo correlación entre ambas variables.

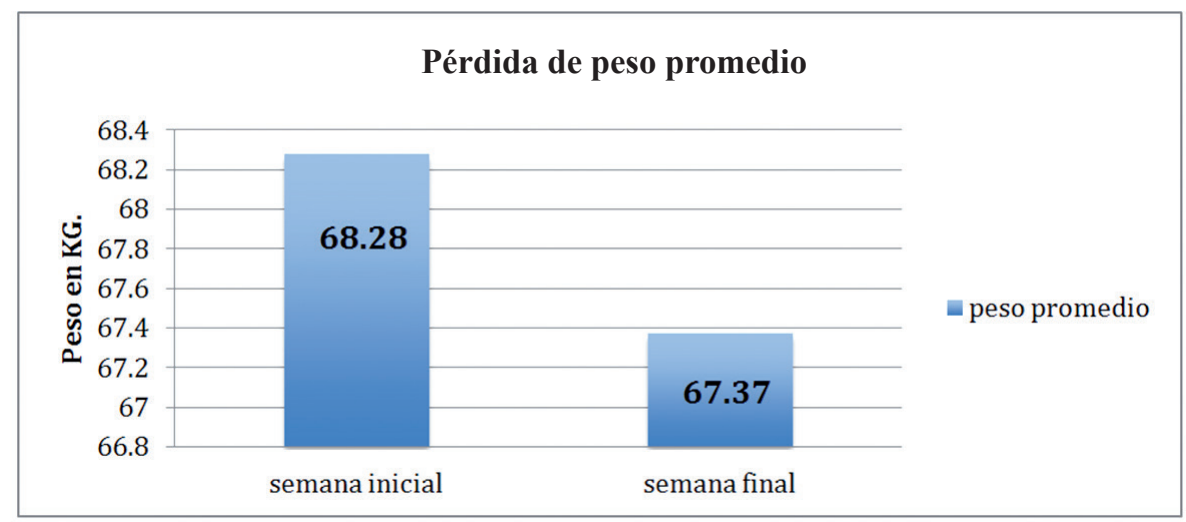

Figura 3

Pérdida de peso promedio

En la pérdida de peso promedio inicial de los participantes fue de $68.28 \mathrm{~kg}$. y su peso final fue de 67.37 kg., encontrándose una disminución de 0.91

\section{DISCUSIÓN}

En las dos primeras semanas se encontró una relación estrecha entre la pérdida de masa grasa y la hidratación, con una significancia de 0,01, y 0,03 siendo muy significativa la relación la prueba que se usó para demostrar (usando el método estadístico de relación y correlación de Pearson).

En el estudio de Hamlet Betancour León y Col (2007), cuyo objetivo fue medir la eficacia de la evaluación antropométrica en la pérdida de masa grasa, y que dichas pérdidas por mínima que parezcan y llevado a escalas prolongadas en el tiempo, sea mayor la significancia, asimismo, en nuestro estudio se encontró la misma significancia de pérdida en las dos primeras semanas.
Kg. en promedio durante el mes de evaluación, según se muestra la figura 3.

Las mediciones de la osmolalidad del plasma y el agua corporal total son actualmente las mejores mediciones de evaluación de la hidratación para estudios de evaluación, a gran escala, de las necesidades de líquidos, no hay actualmente un consenso para la evaluación de la hidratación de una persona, sea que realice actividad física o deporte en competencia, para preferir alguna propuesta de evaluación (Moyano, 1992; Consolazio, 1963).

En contraste a este estudio, para realizar la medición del nivel de hidratación, se decidió utilizar el Test de coloración de la orina con una escala del 1 al 8, por ser de fácil evaluación y adquisición, además de ser aceptable para la persona evaluada. 
La medición de la masa corporal combinado con alguna medición de la concentración de orina en una muestra de la primera orina del día en la mañana, ofrece un método de evaluación sencillo y permite una amplia sensibilidad para detectar desviaciones significativas de la pérdida de masa grasa, para los atletas que entrenan o practican una actividad física, según Consolazio, C.F. Jhnson R.E. Pécora, L. J. (1963).

Un punto a resaltar, en el presente estudio, fue que no se encontraron personas en buen estado de hidratación en la escala de coloración de orina de Armstrong 2000, la gran mayoría se encontraba en una DHT significativa con un $81.25 \%$, en las dos primeras semanas se incrementó la deshidratación en un $10 \%$ y las dos últimas semanas se redujo en un $7.5 \%$, siendo así que la deshidratación fue constante en la gran mayoría de los participantes.

Un caso similar a nuestro estudio fue realizado por H. Betancourt León y Col (2007), en la investigación titulada composición corporal de bailarines élites de la Compañía Ballet Nacional de Cuba, cuyo objetivo fue de evaluar la composición corporal a partir de las mediciones antropométricas, la pérdida de la masa grasa y el incremento de la masa magra, a esta se contrasta un estudio similar sobre la evaluación de la hidratación en atletas por N. Samuel y N. Michael (2005), ambos autores dicen que aunque no hay un consenso científico en el cual se pueda cruzar estas variables para medir la pérdida de masa grasa según el estado dé hidratación, ambos métodos son herramientas para el buen estado de salud y mejora en su rendimiento de las personas en el estudio que practique una disciplina o actividad física.
En conclusión, se quiso saber si la hidratación era una variable que influía directamente en la pérdida de masa grasa. En las dos primeras semanas encontramos una fuerte relación en la pérdida de masa grasa, pero en las dos últimas dos semanas no se encuentra ninguna relación, esto es debido a muchos factores o variables que no se han considerado como: el tipo de dieta, la intensidad de la carga de ejercicio, el descanso, la poca información sobre un régimen de hidratación, entre otros.

\section{CONCLUSIONES}

Se encontró que en las dos primeras semanas de evaluación hubo una influencia significativa y en las dos restantes no hubo influencia, dando a entender que existen otras variables que influyen en la pérdida de masa grasa como la alimentación, la intensidad de ejercicio, género y diferentes tipos de metabolismo.

Entre los participantes, en los resultados referidos al nivel de hidratación, ocurrió en 1.25\% (mínimo), $85 \%$ (significativo) y el $13.8 \%$ (severo) de DHT respectivamente.

Todos los participantes redujeron su porcentaje de masa grasa. Teniendo en cuenta que los hombres tuvieron una disminución de un $-8,7 \%$, a comparación de las mujeres quienes solo disminuyeron $-5.2 \%$ de masa grasa.

Durante todo el mes de evaluación, no se encontró una relación significativa entre el nivel de hidratación y la pérdida de masa grasa, pues existen otras variables que influyen en la pérdida de masa grasa. 
Relación del nivel de hidratación y la pérdida de masa grasa en personas que realizan actividad física en gimnasios de la Urbanización Alameda de Ñaña, Lurigancho Chosica, Lima 2013

\section{REFERENCIAS BIBLIOGRÁFICAS}

Consolazio, C.F., Jhnson R.E. Pecora, L. J. (1963). Las mediciones fisiológicas y funciones metabólicas en el hombre. New York Mc. Grawhill, 389-390.

Consolazio, C.F., Jhonson R.E. Pecora, L. J. (1963). Las mediciones fisiológicas y funciones metabólicas en el hombre. New York Mc. Grawhill . pag. 509-519.

Gil Hernández, Ángel. (2010). Martínez de Victoria Muñoz, Emilio. Tratado de nutrición: nutrición humana en el estado de salud. Madrid: Médica Panamericana.

Hamlet Betancour, León y Colaboradores (2007). Composición Corporal de Bailarines. Élites de la compañía ballet nacional. Cuba.
Moyano V. (1992). Nutrición y suplementos, Editorial Médica Panamericana, 7-8, Buenos Aires Argentina.

N. Samuel y N. Michael (2005). Evaluación de la hidratación en atletas. Revista Científica. Estados Unidos.

Tomas R. (2009), Hacia una definición del sedentarismo. Revista Chilena de Cardiología. Chile.

Universidad Maimónides, (2010). Nivel de hidratación. Argentina: Disponible en: http://weblog.maimonides. edu/nutricion/news_diciembre/news_diciembre.html. 